\title{
Aquaculture
}

\section{Lipid composition and vitamin content of wild female Litopenaeus vannamei in different stages of sexual maturation}

\author{
Roeland Wouters ${ }^{\mathrm{a}, \mathrm{b}, *}$, César Molina ${ }^{\mathrm{a}}$, Patrick Lavens ${ }^{\mathrm{b}}$, \\ Jorge Calderón ${ }^{a}$ \\ ${ }^{a}$ CENAIM-ESPOL Foundation, P.O. Box 0901 4519, Guayaquil, Ecuador \\ ${ }^{\mathrm{b}}$ Laboratory of Aquaculture and Artemia Reference Center, Ghent University, Rozier 44, \\ B-9000 Gent, Belgium
}

Received 11 August 2000; received in revised form 3 January 2001; accepted 4 January 2001

\begin{abstract}
Wild Litopenaeus vannamei females in different stages of sexual maturation were sampled, including spent females and their nauplii, for determination of the lipid content, lipid class (LC) composition, fatty acid (FA) composition, vitamin C content and vitamin E content. Free FA (FFA), triacylglycerol (TAG), phosphatidylcholine (PC) and sterol esters (SE) were the dominant LC in the midgut gland. TAG and phospholipids (PL), mainly PC and phosphatidylethanolamine (PE), were the dominant ovarian LC. Neutral lipids (NL) prevailed over polar lipids (POL) in midgut gland lipids, while ovarian lipids displayed an inverse relationship. An increase in ovarian TL was observed from stage 0 (immature) to stage 1 (early maturing). Later, from stage 1 to stage 2 (mid maturing), a decrease in midgut gland TL was observed. TAG was most responsible for these changes in TL. Lipids were preferentially transferred to the nauplii, which contained relatively high TAG and PC levels. In both midgut gland and ovaries, 16:00, 18:00, 16:1 $n-7$, 18:1 $n-9$, arachidonic acid (ARA; 20:4n-6), eicosapentaenoic acid (EPA; 20:5n-3) and docosahexaenoic acid (DHA; 22:6n-3) were the principal FA. All tissues and nauplii displayed $n-3>n-6$ and EPA $>$ DHA relationships, and contained high proportions of $n-3$ highly unsaturated FA ( $n-3$ HUFA). During sexual maturation, the sum of poly-unsaturated FA (PUFA) decreased in the ovaries due to the decrease in $n-6$ PUFA such as ARA. The sum of mono-unsaturated FA (MUFA), on the other hand, increased in the ovaries. AA levels were high
\end{abstract}

\footnotetext{
${ }^{*}$ Corresponding author. Laboratory of Aquaculture and Artemia Reference Center, Ghent University, Rozier 44, B-9000 Gent, Belgium. Tel.: +32-9-264-37-54; fax: +32-9-264-41-93.

E-mail address: r.wouters@inve.be (R. Wouters).
}

0044-8486/01/\$ - see front matter (C) 2001 Elsevier Science B.V. All rights reserved. PII: S 0044-8486(01)00522-1 
in immature, maturing and mature ovaries. They were low in the ovaries of spent females and nauplii. Vitamin E levels were low in immature ovaries, increased substantially during ovarian maturation, and then decreased again upon spawning. High vitamin E levels were retained in the nauplii. The findings of this study, combined with those reported in related studies, suggest the importance of $n-3$ HUFA for larval development, of vitamin $\mathrm{C}$ for egg development and hatching, and of vitamin $\mathrm{E}$ for ovarian maturation and larval development. These nutrients cannot be synthesised de novo by shrimp, and should be included at high levels in the broodstock diet. (c) 2001 Elsevier Science B.V. All rights reserved.

Keywords: Lipids; Vitamins; Litopenaeus vannamei; Shrimp; Midgut gland; Ovary; Nauplii; Maturation; Lipid

\section{Introduction}

Litopenaeus vannamei is the most important cultivated shrimp of the western hemisphere and is widely distributed along the Pacific coast. The breeding grounds of this species are estuarine areas and bays, which are abundant in Ecuador and Panama. In this region, the shrimp culture industry principally depends on wild postlarvae for stocking grow-out ponds, wild gravid females that produce the nauplii required for stocking the shrimp hatcheries, and wild males and non-gravid females to supply the maturation units of the shrimp hatcheries (Mosquera, 1999). Surprisingly, publications on the biology and fisheries aspects of this species are limited. Information about the biochemical composition exists for wild postlarvae, i.e. fatty acid composition (Montaño and Navarro, 1996), but not for other stages of the life cycle.

Dietary requirements of shrimp are generally higher in sexually maturing adults than in non-reproductive adults and juveniles, but knowledge in this field is still limited (see Harrison, 1990, 1997 for reviews on broodstock nutrition). During maturation, which can take from 3 days to 1 month, the weight of the ovaries increases four to eightfold (Jeckel et al., 1989; Mourente and Rodriguez, 1991; Ravid et al., 1999). Within that time span, sufficient nutrients must be accumulated in the egg yolk to allow the normal development of the embryos and pre-feeding larvae. In several studies, poor reproductive performance or low offspring quality was observed when suboptimal broodstock diets were used (Chamberlain, 1988; Bray et al., 1990; Cahu et al., 1995). The challenge is to increase our knowledge of broodstock nutrition so that large-scale commercial production of high quality nauplii can be achieved. One effective approach is the determination of the biochemical composition of wild shrimp broodstock, and evaluating the variation that occurs during ovarian maturation (Harrison, 1990).

Lipids are an indispensable source of energy and may also be essential nutrients that can only be synthesised de novo by shrimp to a very limited extent, e.g. highly unsaturated fatty acids (HUFA), or cannot be synthesised de novo, e.g. sterols (Chang and O'Connor, 1983; D'Abramo, 1997). The lipid composition of shrimp broodstock has been studied in Farfantepenaeus aztecus (Castille and Lawrence, 1989), F. duorarum (Gehring, 1974), Fenneropenaeus indicus (Read and Caulton, 1980; Galois, 1984), L. setiferus (Middleditch et al., 1980; Castille and Lawrence, 1989), Marsupenaeus japonicus (Teshima and Kanazawa, 1983; Teshima et al., 1989), Melicertus kerathurus 
(Mourente and Rodriguez, 1991), Penaeus monodon (Millamena and Pascual, 1990), P. semisulcatus (Ravid et al., 1999) and Pleoticus muelleri (Jeckel et al., 1989). While many similarities exist among shrimp species, differences in fatty acid (FA) and lipid class (LC) concentrations were observed.

Vitamin requirements for shrimp broodstock are not currently defined, but some studies have shown that dietary vitamins $\mathrm{C}$ and $\mathrm{E}$ improve shrimp reproductive performance (Chamberlain, 1988; Wouters et al., 1999) and egg hatching rate (Cahu et al., 1991, 1995). Alava et al. (1993b) observed retarded ovarian development in $M$. japonicus when vitamins $\mathrm{A}, \mathrm{C}$ and $\mathrm{E}$ were not included in their diet. The content of these vitamins in wild shrimp spawners has yet to be studied.

The present study is an investigation of the lipid content, fatty acid and lipid class composition of the total lipids in the midgut gland and ovaries of maturing and spent $L$. vannamei spawners from the wild. Levels of vitamins $\mathrm{C}$ and $\mathrm{E}$ in the ovary were also determined. To assist in developing a better understanding of nutrient transfer from females to offspring, all these biochemical parameters were also determined in the nauplii from wild spawners. This work complements similar studies on wild Macrobachium rosenbergii tissues conducted by our laboratory at the Ghent University (Cavalli, 2000).

\section{Materials and methods}

\subsection{Sampling}

Female L. vannamei of $61.67 \pm 12.83 \mathrm{~g}(N=70)$ representing different stages of sexual maturation were purchased from artesanal fishermen on the beach of Tonchigüe (Esmeraldas Province, Ecuador). Gravid females were transferred to a nearby hatchery and placed in individual spawning tanks. The next day, eggs hatched in the same tanks and nauplii were harvested and rinsed with fresh water. All animals were placed on dry ice immediately for transport to the research facilities of the CENAIM-ESPOL Foundation in San Pedro de Manglaralto (Guayas Province, Ecuador), where they where stored at $-80^{\circ} \mathrm{C}$. The females were visually examined and then grouped according to the stage of sexual maturation: stage 0, immature (thin and transluscent ovaries, not visible through the exoskeleton); stage 1, early maturing (white-yellow ovaries, the middle lobes are not visible); stage 2, mid-maturing (yellow-gray ovaries, anterior and posterior lobes have a greater diameter than that in the preceding stage); stage 3, late maturing (orange ovaries, mid lobes become visible); stage 4, fully mature (red-green ovaries, large anterior, posterior and mid lobes); spent female (ovaries are indistinguishable from stage 0 ovaries after spawning). Animals were dissected on ice and the midgut gland and ovaries were excised. Wet weight (WW) of intact females and these organs were determined to allow calculation of the gonadosomatic index $(\mathrm{GSI}=100 \times$ ovary $\mathrm{WW} /$ animal $\mathrm{WW})$ and the midgut gland index $(\mathrm{MGI}=100 \times$ midgut gland $\mathrm{WW}$ /animal WW). The tissues were pooled to obtain a sufficient amount of sample to conduct lipid and vitamin analyses. The availability of females in different stages was a 
limiting factor. Three samples were collected for the tissues of females in stage 0 (six females per pool), stage 1 (six females per pool), stage 2 (four females per pool)and stage 4 (two females per pool). Two samples were collected for the females in stage 3 ( 2 females per pool) and for spent females (six females per pool). Nauplii collected from three gravid wild females were not pooled.

\subsection{Sample processing and biochemical analyses}

Midgut gland and ovarian tissue was homogenized on ice. Nauplii were kept intact. Samples were divided into subsamples and stored in dark glass vials that were nitrogen-flushed and sealed with Teflon ${ }^{\circledR}$ screw caps. The sample vials were then transported in a styrofoam box with dry ice to the Ghent University (Belgium) for lipid and vitamin analyses.

Moisture of triplicate samples was determined by drying in an oven at $60^{\circ} \mathrm{C}$ for $24 \mathrm{~h}$. Triplicate biochemical analyses were conducted according to the following standard procedures. Total lipids (TL) were extracted according to the method described by Folch et al. (1957) as modified by Ways and Hanahan (1964). FA composition was analytically verified by flame ionization detection (FID) after injecting the sample into a Chrompack CP9001 gas chromatograph according to the procedure described by Coutteau and Sorgeloos (1995). LC were determined by means of high performance thin-layer chromatography (HPTLC) according to the procedure reported by Olsen and Henderson (1989). Vitamin C (AA, ascorbic acid) concentrations were analyzed by means of high performance liquid chromatography (HPLC) as described by Nelis et al. (1997). Vitamin E ( $\alpha$-tocopherol and $\gamma$-tocopherol) were analyzed using HPLC according to the procedure described by Huo et al. (1999).

\subsection{Statistical analyses}

Results were expressed as $\%$ of total FA and $\mathrm{mg} \mathrm{g}^{-1}$ dry matter (DM) for FA, \% of $\mathrm{DM}$ for TL, \% of TL for LC, and $\mathrm{mg} \mathrm{kg}^{-1} \mathrm{DM}$ for vitamins. The limited number of subsamples analyzed precluded testing for the normality of these data (Castell et al., 1995). None of the percent values approached $100 \%$ and none of those for individual LC or FA surpassed $35 \%$, for which no data transformation was done. Homogeneity of variances was verified with the Levene test. The assumption of homogeneity of variances was fulfilled in the majority of the cases. However, whenever failure of this assumptions was observed, the changes due to ovarian maturation or spawning were small compared to the average value and thus the assumption was near enough to the truth for the analysis to proceed (Mead et al., 1993). Analysis of variance (one-way ANOVA) was used to determine statistical differences between different stages of sexual maturation. Nauplii were not included in the statistical analyses. The Duncan test was performed for multiple comparison. All references to significant differences are at the 5\% level or lower. The amount of sample from the stage 3 ovary was insufficient to conduct quantitative FA (for which quantitative FA data were not submitted to statistical analysis) and vitamin $\mathrm{E}$ determinations. 


\section{Results}

\subsection{Tissue indices and dry matter content}

Table 1 presents the tissue indices and DM content of the midgut glands and ovaries of spawners classified according to the stage of sexual maturation. Changes in GSI during ovarian maturation were very pronounced, and a ninefold increase in ovarian weight occurred during maturation. Ovarian DM increased from $19.4 \%$ to $31.0 \%$ during maturation. The DM content of wild nauplii was $18.8 \pm 0.2 \%$.

\subsection{Lipid classes during ovarian maturation and after spawning}

TL content and LC composition of the midgut gland are presented in Table 2. Those of ovaries and nauplii are presented in Table 3. TL levels in the midgut gland decreased from stage $1(35.2 \%)$ to stage $2(21.0 \%)$ and did not significantly differ throughout the remaining stages of maturation and after spawning. Ovarian TL, in contrast, increased dramatically at the very first onset of ovarian maturation (8.6\% in stage 0 vs. $21.7 \%$ in stage 1) and did not significantly differ through stage 4 . In spent ovaries the TL level dropped to a level that was not significantly different from that of immature ovaries. Lipids seemed to be efficiently transferred to the nauplii, because they contained a record level of $27 \%$, considerably higher than in fully mature ovaries.

Lipid class analyses revealed that the midgut gland contained two to four times more neutral lipids (NL) than polar lipids (POL). Free FA (FFA), triacylglycerol (TAG), phosphatidylcholine (PC) and sterol esters (SE) were identified as the main lipid components of the midgut gland. The group cholesterol + diacylglycerols + pigments $(\mathrm{COL}+\mathrm{DAG}+\mathrm{PIGM})$ also composed a relatively high proportion of the total lipids. The decrease of TL from stage 1 to stage 2 in the midgut gland seems to be principally

Table 1

Midgut gland index (MGI), gonadosomatic index (GSI) and dry matter content (DM; \%) in midgut glands and ovaries of female L. vannamei shrimp in different stages of sexual maturation

\begin{tabular}{|c|c|c|c|c|c|c|}
\hline \multirow[b]{3}{*}{$N$} & \multicolumn{6}{|l|}{ Stage } \\
\hline & 0 & 1 & 2 & 3 & 4 & SF \\
\hline & 18 & 18 & 12 & 4 & 6 & 12 \\
\hline \multicolumn{7}{|c|}{ Midgut gland } \\
\hline MGI & $2.8^{\mathrm{b}}$ & $2.5^{\mathrm{b}}$ & $2.4^{\mathrm{b}}$ & $1.7^{\mathrm{a}}$ & $2.1^{\mathrm{ab}}$ & $2.3^{\mathrm{b}}$ \\
\hline $\mathrm{DM}$ & $34.5^{\mathrm{ab}}$ & $36.0^{\mathrm{b}}$ & $32.5^{\mathrm{ab}}$ & $32.6^{\mathrm{ab}}$ & $33.8^{\mathrm{ab}}$ & $29.8^{\mathrm{a}}$ \\
\hline \multicolumn{7}{|c|}{ Ovary } \\
\hline GSI & $1.0^{\mathrm{a}}$ & $2.0^{\mathrm{b}}$ & $5.6^{\mathrm{c}}$ & $7.2^{\mathrm{d}}$ & $9.0^{\mathrm{e}}$ & $2.3^{\mathrm{b}}$ \\
\hline DM & $19.4^{\mathrm{a}}$ & $27.6^{\mathrm{bc}}$ & $31.4^{\mathrm{cd}}$ & $26.3^{\text {bcd }}$ & $31.0^{\mathrm{d}}$ & $23.5^{\mathrm{b}}$ \\
\hline
\end{tabular}

Data represent mean values. Within a row, values with different superscript letters are significantly different $(P \leq 0.05)$ 
Table 2

Total lipids (TL; \% of dry matter) and lipid class composition (\% of TL) in midgut glands of L. vannamei in different stages of sexual maturation

\begin{tabular}{|c|c|c|c|c|c|c|}
\hline & \multicolumn{6}{|l|}{ Stage } \\
\hline & 0 & 1 & 2 & 3 & 4 & SF \\
\hline \multicolumn{7}{|l|}{ Polar lipids $(P O L)$} \\
\hline LPC & $2.7^{\mathrm{a}}$ & $3.1^{\mathrm{ab}}$ & $4.7^{\mathrm{cd}}$ & $5.9^{\mathrm{d}}$ & $4.2^{\mathrm{bc}}$ & $4.2^{\mathrm{bc}}$ \\
\hline SM & $0.5^{\mathrm{ab}}$ & $0.4^{\mathrm{a}}$ & $0.7^{\mathrm{b}}$ & $1.1^{\mathrm{c}}$ & $0.7^{\mathrm{b}}$ & $0.4^{\mathrm{ab}}$ \\
\hline $\mathrm{PC}$ & $9.6^{\mathrm{a}}$ & $10.6^{\mathrm{a}}$ & $12.0^{\mathrm{ab}}$ & $12.2^{\mathrm{ab}}$ & $13.6^{\mathrm{b}}$ & $11.2^{\mathrm{ab}}$ \\
\hline PS & $1.6^{\mathrm{b}}$ & $0.6^{\mathrm{a}}$ & $3.4^{\mathrm{c}}$ & $3.6^{\mathrm{c}}$ & $2.6^{\mathrm{c}}$ & $3.1^{\mathrm{c}}$ \\
\hline PI & $1.0^{\mathrm{ab}}$ & $0.2^{\mathrm{a}}$ & $1.1^{\mathrm{ab}}$ & $0.8^{\mathrm{ab}}$ & $1.5^{\mathrm{b}}$ & $1.6^{\mathrm{b}}$ \\
\hline PA & $1.6^{\mathrm{a}}$ & $1.0^{\mathrm{a}}$ & $1.8^{\mathrm{ab}}$ & $1.7^{\mathrm{ab}}$ & $2.7^{\mathrm{c}}$ & $2.5^{\mathrm{bc}}$ \\
\hline PE & $4.6^{\mathrm{ab}}$ & $3.0^{\mathrm{a}}$ & $6.3^{b c}$ & $7.0^{\mathrm{c}}$ & $7.2^{\mathrm{c}}$ & $6.6^{\mathrm{bc}}$ \\
\hline DGDG & $0.3^{\mathrm{ab}}$ & $0.0^{\mathrm{a}}$ & $1.5^{\mathrm{c}}$ & $1.1^{\mathrm{c}}$ & $1.0^{\mathrm{c}}$ & $0.8^{\mathrm{bc}}$ \\
\hline$?$ & 0.0 & 0.0 & 0.2 & 1.2 & 0.0 & 0.6 \\
\hline MGDG & $0.0^{\mathrm{a}}$ & $0.8^{\mathrm{b}}$ & $0.1^{\mathrm{a}}$ & $0.0^{\mathrm{a}}$ & $0.0^{\mathrm{a}}$ & $0.1^{\mathrm{a}}$ \\
\hline \multicolumn{7}{|l|}{ Neutral lipids (NL) } \\
\hline MAG + PIGM & $6.8^{\mathrm{a}}$ & $9.6^{\mathrm{b}}$ & $9.6^{\mathrm{b}}$ & $8.7^{b}$ & $7.5^{\mathrm{ab}}$ & $9.6^{\mathrm{b}}$ \\
\hline $\mathrm{COL}+\mathrm{DAG}+\mathrm{PIGM}$ & $15.8^{\mathrm{bc}}$ & $19.8^{d}$ & $16.8^{\mathrm{bc}}$ & $13.5^{\mathrm{a}}$ & $15.2^{\mathrm{ab}}$ & $17.5^{\mathrm{c}}$ \\
\hline FFA & $20.5^{\mathrm{a}}$ & $24.2^{\mathrm{b}}$ & $28.2^{\mathrm{c}}$ & $27.6^{\mathrm{c}}$ & $22.5^{\mathrm{ab}}$ & $27.9^{c}$ \\
\hline TAG & $23.0^{\mathrm{b}}$ & $16.7^{\mathrm{ab}}$ & $8.0^{\mathrm{a}}$ & $9.4^{\mathrm{a}}$ & $8.0^{\mathrm{a}}$ & $8.0^{\mathrm{a}}$ \\
\hline SE & $10.6^{b}$ & $7.9^{\mathrm{ab}}$ & $5.9^{\mathrm{a}}$ & $6.5^{\mathrm{ab}}$ & $9.8^{a b}$ & $5.7^{\mathrm{a}}$ \\
\hline TL & $36.3^{b}$ & $35.2^{\mathrm{b}}$ & $21.0^{\mathrm{a}}$ & $16.7^{\mathrm{a}}$ & $21.5^{\mathrm{a}}$ & $24.6^{\mathrm{a}}$ \\
\hline Sum POL & $23.3^{\mathrm{a}}$ & $19.7^{\mathrm{a}}$ & $31.5^{\mathrm{b}}$ & $34.2^{\mathrm{b}}$ & $33.4^{\mathrm{b}}$ & $31.2^{\mathrm{b}}$ \\
\hline Sum NL & $76.7^{\mathrm{b}}$ & $80.3^{\mathrm{b}}$ & $68.5^{\mathrm{a}}$ & $65.8^{\mathrm{a}}$ & $66.6^{\mathrm{a}}$ & $68.8^{\mathrm{a}}$ \\
\hline $\mathrm{POL} / \mathrm{NL}$ & $0.30^{\mathrm{ab}}$ & $0.25^{\mathrm{a}}$ & $0.46^{\mathrm{bc}}$ & $0.52^{\mathrm{c}}$ & $0.51^{\mathrm{c}}$ & $0.45^{\mathrm{bc}}$ \\
\hline
\end{tabular}

Data represent mean values. Within a row, values with different superscript letters are significantly different $(P \leq 0.05)$.

Abbreviations: COL, cholesterol; DAG, diacylglycerol; DGDG, digalactosyldiacylglycerol; FA, fatty acid; LPC, lyso-phosphatidylcholine; MAG, monoacylglycerol; MGDG, monogalactosyldiacylglycerol; NL, neutral lipid; PA, phosphatic acid; PC, phosphatidylcholine; PE, phosphatidylethanolamine; PI, phophatidylinositol; PIGM, pigments; PL, phospholipid; POL; polar lipid; PS, phophatidylserine; PUFA, polyunsaturated fatty acid; SE, sterol esters; SF, spent females; SM, sphingomyelin; TAG, triacylglycerol; TL, total lipid.

the result of a decrease in TAG (although the decrease in TAG is not statistically significant). Other NL revealed up and down trends. The POL of the midgut gland increased significantly from stage 1 to stage 2, due to the increase in several individual phospholipids (PL) and digalactosyldiacylglycerol (DGDG).

Initially, ovarian lipids were composed of more POL than NL, but as sexual maturation proceeded, ovarian POL decreased and ovarian NL increased to a point when the POL/NL ratio of fully mature females was 0.97. PC, TAG and phosphatidylethanolamine (PE) were the predominant ovarian lipid classes. The increase in TAG during maturation was principally responsible for the increase in ovarian TL from stage 0 to stage 1 . FFA and the group COL + DAG + PIGM decreased at the onset of ovarian maturation. Significant decreases in levels of phosphatidylserine (PS), phosphatidylinositol (PI), phosphatic acid (PA) and PE were observed. PC increased from 
Table 3

Total lipids (TL; \% of dry matter) and lipid class composition (\% of TL) in ovaries of L. vannamei according to the sexual maturation stage, and in nauplii

\begin{tabular}{|c|c|c|c|c|c|c|c|}
\hline & \multicolumn{6}{|l|}{ Stage } & \multirow[t]{2}{*}{ Nauplii } \\
\hline & $\overline{0}$ & 1 & 2 & 3 & 4 & $\mathrm{SF}$ & \\
\hline \multicolumn{8}{|l|}{ Polar lipids $(P O L)$} \\
\hline LPC & $2.6^{\mathrm{c}}$ & $0.3^{\mathrm{ab}}$ & $0.1^{\mathrm{ab}}$ & $0.7^{\mathrm{b}}$ & $0.2^{\mathrm{ab}}$ & $0.0^{\mathrm{a}}$ & 1.2 \\
\hline SM & $0.0^{\mathrm{a}}$ & $1.6^{\mathrm{b}}$ & $0.0^{\mathrm{a}}$ & $0.0^{\mathrm{a}}$ & $0.0^{\mathrm{a}}$ & $0.0^{\mathrm{a}}$ & 1.4 \\
\hline $\mathrm{PC}$ & $31.6^{\mathrm{b}}$ & $42.2^{\mathrm{d}}$ & $33.5^{\mathrm{bc}}$ & $34.9^{c}$ & $32.2^{\mathrm{b}}$ & $27.5^{\mathrm{a}}$ & 29.5 \\
\hline PS & $4.1^{\mathrm{c}}$ & $0.5^{\mathrm{a}}$ & $1.3^{\mathrm{b}}$ & $1.7^{\mathrm{b}}$ & $1.7^{\mathrm{b}}$ & $1.6^{\mathrm{b}}$ & 2.1 \\
\hline PI & $5.8^{\mathrm{c}}$ & $2.2^{\mathrm{a}}$ & $3.1^{\mathrm{ab}}$ & $2.8^{\mathrm{ab}}$ & $3.1^{\mathrm{ab}}$ & $3.7^{\mathrm{b}}$ & 2.8 \\
\hline PA & $5.6^{\mathrm{c}}$ & $2.2^{\mathrm{a}}$ & $2.2^{\mathrm{a}}$ & $2.3^{\mathrm{a}}$ & $2.3^{\mathrm{a}}$ & $4.3^{\mathrm{b}}$ & 2.0 \\
\hline PE & $14.0^{\mathrm{c}}$ & $10.1^{\mathrm{a}}$ & $9.6^{\mathrm{a}}$ & $9.4^{\mathrm{a}}$ & $9.0^{\mathrm{a}}$ & $13.3^{\mathrm{b}}$ & 8.8 \\
\hline DGDG & $0.0^{\mathrm{a}}$ & $0.3^{\mathrm{ab}}$ & $0.8^{c}$ & $0.3^{\mathrm{b}}$ & $0.0^{\mathrm{a}}$ & $0.0^{\mathrm{a}}$ & 0.0 \\
\hline$?$ & $0.0^{\mathrm{a}}$ & $0.3^{\mathrm{ab}}$ & $0.2^{\mathrm{ab}}$ & $0.2^{\mathrm{ab}}$ & $0.6^{\mathrm{b}}$ & $0.0^{\mathrm{a}}$ & 0.0 \\
\hline MGDG & $0.8^{\mathrm{c}}$ & $0.0^{\mathrm{a}}$ & $0.7^{\mathrm{b}}$ & $0.7^{\mathrm{bc}}$ & $0.0^{\mathrm{a}}$ & $0.6^{\mathrm{b}}$ & $0.9^{\mathrm{d}}$ \\
\hline \multicolumn{8}{|l|}{ Neutral lipids (NL) } \\
\hline MAG + PIGM & $2.7^{\mathrm{ab}}$ & $1.9^{\mathrm{a}}$ & $2.5^{\mathrm{ab}}$ & $2.3^{\mathrm{ab}}$ & $3.5^{\mathrm{bc}}$ & $4.4^{\mathrm{c}}$ & $1.4^{\mathrm{a}}$ \\
\hline $\mathrm{COL}+\mathrm{DAG}+\mathrm{PIGM}$ & $12.8^{\mathrm{c}}$ & $8.5^{\mathrm{ab}}$ & $9.7^{\mathrm{b}}$ & $7.9^{\mathrm{a}}$ & $8.2^{\mathrm{ab}}$ & $12.8^{\mathrm{c}}$ & 7.9 \\
\hline FFA & $7.4^{\mathrm{b}}$ & $2.0^{\mathrm{a}}$ & $2.5^{\mathrm{a}}$ & $1.7^{\mathrm{a}}$ & $2.3^{\mathrm{a}}$ & $8.7^{\mathrm{c}}$ & 3.3 \\
\hline TAG & $8.3^{\mathrm{a}}$ & $28.1^{\mathrm{c}}$ & $28.7^{\mathrm{c}}$ & $30.2^{\mathrm{c}}$ & $33.8^{c}$ & $20.6^{\mathrm{b}}$ & 33.5 \\
\hline ES & 4.1 & 0.0 & 4.8 & 4.9 & 2.9 & 2.4 & 5.1 \\
\hline TL & $8.6^{\mathrm{a}}$ & $21.7^{\mathrm{bc}}$ & $21.4^{\mathrm{bc}}$ & $21.1^{\mathrm{bc}}$ & $17.2^{\mathrm{b}}$ & $11.7^{\mathrm{ab}}$ & 27.6 \\
\hline Sum POL & $64.5^{c}$ & $59.6^{\mathrm{b}}$ & $51.5^{\mathrm{a}}$ & $53.1^{\mathrm{a}}$ & $49.2^{\mathrm{a}}$ & $51.0^{\mathrm{a}}$ & 48.8 \\
\hline Sum NL & $35.5^{\mathrm{a}}$ & $40.4^{\mathrm{b}}$ & $48.5^{\mathrm{c}}$ & $46.9^{c}$ & $50.8^{\mathrm{c}}$ & $49.0^{\mathrm{c}}$ & 51.2 \\
\hline $\mathrm{POL} / \mathrm{NL}$ & $1.83^{\mathrm{c}}$ & $1.49^{\mathrm{b}}$ & $1.06^{\mathrm{a}}$ & $1.13^{\mathrm{a}}$ & $0.97^{\mathrm{a}}$ & $1.04^{\mathrm{a}}$ & 0.95 \\
\hline
\end{tabular}

Data represent mean values. Within a row, values with different superscript letters are significantly different $(P \leq 0.05)$.

Refer to the previous table for abbreviations.

stage 0 to stage 1 , then decreased from stage 1 to stage 2 . Spent ovaries contained levels of PC and TAG that were lower than those of fully mature ovaries. Levels of PA, PE, FFA and COL + DAG + PIGM in spent ovaries were higher than those of fully mature ovaries. The naupliar LC composition was similar to that of mature ovaries, with the exception of PC, LPC, SM and MAG + PIGM whose concentrations were lower in nauplii.

\subsection{Fatty acid composition during ovarian maturation and after spawning}

The FA composition of the midgut gland is presented in Table 4 . The main fatty acids were 16:0, 16:1 $n-7,18: 0,18: 1 n-7,18: 1 n-9$, arachidonic acid (ARA; 20:4n-6), eicosapentanoic acid (EPA; 20:5n-3) and docosahexaenoic acid (DHA; $22: 6 n-3)$. Approximately $20 \%$ of the FA was $n-3$ HUFA. The midgut gland contained almost $2 \times$ more $n-3$ FA than $n-6$ FA. Only minor changes in individual FA percentages occurred during ovarian maturation. The levels of 15:0 and 17:0 of the midgut gland moved and were finally lower in stage 4 as compared to stage 0 . The proportion of saturated FA (SFA) remained roughly stable. Individual mono-unsaturated 
Table 4

Fatty acid (FA) composition (in $\%$ of total FA and $\mathrm{mg} \mathrm{g}^{-1}$ dry weight) in midgut glands of L. vannamei in different stages of sexual maturation

\begin{tabular}{|c|c|c|c|c|c|c|c|c|c|c|c|c|}
\hline & \multicolumn{12}{|l|}{ Stage } \\
\hline & \multicolumn{2}{|l|}{0} & \multicolumn{2}{|l|}{1} & \multicolumn{2}{|l|}{2} & \multicolumn{2}{|l|}{3} & \multicolumn{2}{|l|}{4} & \multicolumn{2}{|l|}{ SF } \\
\hline & $\%$ & $\begin{array}{l}\mathrm{mg} \\
\mathrm{g}^{-1}\end{array}$ & $\%$ & $\begin{array}{l}\mathrm{mg} \\
\mathrm{g}^{-1}\end{array}$ & $\%$ & $\begin{array}{l}\mathrm{mg} \\
\mathrm{g}^{-1}\end{array}$ & $\%$ & $\begin{array}{l}\mathrm{mg} \\
\mathrm{g}^{-1}\end{array}$ & $\%$ & $\begin{array}{l}\mathrm{mg} \\
\mathrm{g}^{-1}\end{array}$ & $\%$ & $\begin{array}{l}\mathrm{mg} \\
\mathrm{g}^{-1}\end{array}$ \\
\hline 14:0 & 3.9 & 24.5 & 4.6 & 31.8 & 4.3 & 29.3 & 4.0 & 24.9 & 3.5 & 19.4 & 4.3 & 27.6 \\
\hline $15: 0$ & $1.9^{\mathrm{bc}}$ & 12.3 & $2.2^{c}$ & 15.3 & $1.8^{\mathrm{b}}$ & 11.8 & $1.7^{\mathrm{b}}$ & 13.8 & $1.4^{\mathrm{a}}$ & 7.6 & $1.2^{\mathrm{a}}$ & 8.1 \\
\hline 16:0 & 18.8 & 119.3 & 21.7 & 149.7 & 20.8 & 140.1 & 20.5 & 140.0 & 18.2 & 100.1 & 21.1 & 133.7 \\
\hline $16: 1 n-7$ & 5.2 & 32.9 & 5.8 & 40.1 & 6.6 & 44.4 & 6.9 & 43.6 & 7.0 & 38.2 & 7.0 & 44.8 \\
\hline $17: 0$ & $2.1^{\mathrm{d}}$ & 13.3 & $2.0^{\mathrm{cd}}$ & 13.7 & $1.7^{b c}$ & 11.5 & $1.9^{\mathrm{cd}}$ & 15.8 & $1.6^{\mathrm{ab}}$ & 8.7 & $1.4^{\mathrm{a}}$ & 7.6 \\
\hline 18:0 & 8.9 & 56.6 & 8.4 & 58.0 & 8.1 & 54.3 & 8.4 & 69.0 & 7.7 & 42.4 & 7.5 & 48.1 \\
\hline $18: 1 n-9$ & 5.3 & 33.3 & 6.2 & 42.8 & 6.4 & 42.9 & 5.9 & 40.5 & 6.8 & 37.6 & 6.3 & 39.8 \\
\hline $18: 1 n-7$ & 5.0 & 31.9 & 5.3 & 36.3 & 5.4 & 36.0 & 5.3 & 37.1 & 5.0 & 27.2 & 5.6 & 36.1 \\
\hline $20: 1 n-9$ & 1.0 & 6.2 & 0.9 & 6.1 & 0.9 & 5.7 & 0.7 & 5.6 & 1.0 & 5.3 & 0.9 & 6.3 \\
\hline $20: 1 n-7$ & 1.7 & 11.0 & 1.7 & 11.5 & 1.4 & 9.5 & 1.1 & 8.4 & 1.3 & 7.1 & 1.7 & 11.0 \\
\hline $20: 2 n-6$ & 1.4 & 8.5 & 1.3 & 9.0 & 1.1 & 7.3 & 1.1 & 9.2 & 1.1 & 6.3 & 1.1 & 7.3 \\
\hline $20: 4 n-6$ & 5.3 & 33.9 & 5.1 & 35.2 & 5.1 & 34.2 & 6.1 & 54.7 & 5.8 & 31.7 & 5.0 & 32.0 \\
\hline $20: 5 n-3$ & 9.7 & 61.6 & 10.0 & 71.6 & 10.1 & 67.2 & 10.3 & 84.5 & 10.5 & 57.2 & 11.9 & 75.5 \\
\hline $22: 4 n-6$ & $2.5^{\mathrm{c}}$ & 16.1 & $1.9^{\mathrm{ab}}$ & 13.2 & $2.1^{\mathrm{abc}}$ & 14.2 & $1.9^{a b c}$ & 18.4 & $2.4^{\mathrm{bc}}$ & 13.3 & $1.6^{\mathrm{a}}$ & 10.4 \\
\hline $22: 5 n-6$ & $1.3^{\mathrm{c}}$ & 8.3 & $1.1^{\mathrm{bc}}$ & 7.2 & $1.0^{\mathrm{ab}}$ & 6.3 & $0.8^{\mathrm{a}}$ & 6.9 & $1.2^{\mathrm{c}}$ & 6.7 & $0.8^{\mathrm{a}}$ & 5.8 \\
\hline $22: 5 n-3$ & $2.7^{b}$ & 16.9 & $2.0^{\mathrm{ab}}$ & 13.8 & $2.1^{\mathrm{ab}}$ & 14.0 & $1.8^{\mathrm{a}}$ & 16.9 & $2.2^{\mathrm{ab}}$ & 12.1 & $1.7^{\mathrm{a}}$ & 11.0 \\
\hline $22: 6 n-3$ & $8.3^{\mathrm{ab}}$ & 52.5 & $8.6^{\mathrm{b}}$ & 59.4 & $6.8^{\mathrm{ab}}$ & 45.1 & $6.5^{\mathrm{a}}$ & 57.5 & $7.9^{\mathrm{ab}}$ & 43.0 & $6.6^{\mathrm{a}}$ & 41.7 \\
\hline SFA & 37.6 & 238.9 & 39.6 & 272.7 & 37.3 & 251.4 & 37.8 & 267.4 & 34.1 & 187.2 & 36.3 & 229.1 \\
\hline MUFA & $20.5^{\mathrm{a}}$ & 129.7 & $22.4^{\mathrm{ab}}$ & 155.1 & $23.4^{\mathrm{ab}}$ & 156.8 & $22.6^{\mathrm{ab}}$ & 157.6 & $24.8^{\mathrm{b}}$ & 136.0 & $24.0^{\mathrm{ab}}$ & 153.1 \\
\hline PUFA & 37.4 & 219.9 & 30.2 & 210.2 & 31.8 & 210.2 & 31.8 & 269.2 & 34.3 & 187.6 & 32.5 & 207.5 \\
\hline$n-3$ & 22.1 & 138.0 & 19.1 & 131.8 & 20.8 & 136.0 & 20.1 & 168.1 & 21.7 & 117.3 & 22.2 & 138.5 \\
\hline$n-6$ & 12.6 & 80.0 & 11.1 & 76.6 & 11.0 & 72.5 & 11.6 & 99.9 & 12.6 & 69.1 & 10.3 & 66.3 \\
\hline $\begin{array}{l}n-3 \\
\text { HUFA }\end{array}$ & 21.6 & 136.4 & 17.9 & 125.4 & 19.7 & 130.2 & 13.2 & 162.4 & 21.2 & 115.9 & 21.0 & 133.5 \\
\hline $\begin{array}{l}n-3 / \\
n-6\end{array}$ & 1.76 & & 1.74 & & 1.89 & & 1.73 & & 1.72 & & 2.14 & \\
\hline $\begin{array}{l}\text { DHA/ } \\
\text { EPA }\end{array}$ & $0.85^{\mathrm{c}}$ & & $0.82^{c}$ & & $0.67^{\mathrm{ab}}$ & & $0.6^{a b}$ & & $0.75^{b c}$ & & $0.56^{\mathrm{a}}$ & \\
\hline
\end{tabular}

Data represent mean values. Sums include FA present in minor concentrations not listed in the table Within a row, \% values with different superscript letters are significantly different $(P \leq 0.05)$.

Abbreviations: DHA, docosahexaenoic acid (22:6n-3); EPA, eicosapentaenoic acid (20:5n-3); FA, fatty acid; HUFA, highly unsaturated fatty acid; MUFA, monounsaturated fatty acid; PUFA, polyunsaturated fatty acid; SFA, saturated fatty acid; SF, spent females.

FA (MUFA) did not change during maturation. However, the sum of MUFA was significantly higher in stage 4 than in stage 0 . Some fluctuation was observed in the percentages of 22:4(n-6), 22:5n-6,22:5n-3 and DHA, though similar levels were found for them in stage 0 and stage 4 of the midgut gland. The sum of polyunsaturated FA (PUFA) and $n-3$ HUFA remained stable throughout ovarian maturation. The DHA/EPA ratio decreased from stage 1 to stage 2. A post-spawning reduction in $n-6$ FA and the ratio DHA/EPA occurred. 
The FA compositions of ovaries and nauplii (Table 5) were similar to those of the midgut gland, wherein the same predominant FA, a high $n-3$ HUFA content and a high $n-3 / n-6$ ratio were observed. However, $n-3 / n-6$ ratios in the ovaries were higher than those of the midgut gland. The levels of many of the individual ovarian FA changed significantly during sexual maturation. A moderate increase in 14:0 and 16:0 were observed, while 15:0 revealed up and down trends. The total percentage of SFA did not change during maturation. 16:1n-7 increased, 18:1n-9 decreased, and 18:1 $n-7$ fluctuated. In total, MUFA increased, 22:4n-6 increased, ARA decreased, and 22:5n-6 fluctuated. The total percentage of PUFA decreased slightly with maturation due to a decrease in $n-6$ FA. $n-3$ FA and the sum of $n-3$ HUFA

Table 5

Fatty acid (FA) composition (in \% of total FA and $\mathrm{mg} \mathrm{g}^{-1} \mathrm{DW}$ ) in ovaries of L. vannamei in different stages of sexual maturation, and in nauplii

\begin{tabular}{|c|c|c|c|c|c|c|c|c|c|c|c|c|c|c|}
\hline & \multicolumn{12}{|l|}{ Stage } & \multicolumn{2}{|c|}{ Nauplii } \\
\hline & \multicolumn{2}{|l|}{0} & \multicolumn{2}{|l|}{1} & \multicolumn{2}{|l|}{2} & \multicolumn{2}{|l|}{3} & \multicolumn{2}{|l|}{4} & \multicolumn{2}{|l|}{ SF } & \multirow[b]{2}{*}{$\%$} & \multirow[b]{2}{*}{$\begin{array}{l}\mathrm{mg} \\
\mathrm{g}^{-1}\end{array}$} \\
\hline & $\%$ & $\begin{array}{l}\mathrm{mg} \\
\mathrm{g}^{-1}\end{array}$ & $\%$ & $\begin{array}{l}\mathrm{mg} \\
\mathrm{g}^{-1}\end{array}$ & $\%$ & $\begin{array}{l}\mathrm{mg} \\
\mathrm{g}^{-1}\end{array}$ & $\%$ & $\begin{array}{l}\mathrm{mg} \\
\mathrm{g}^{-1}\end{array}$ & $\%$ & $\begin{array}{l}\mathrm{mg} \\
\mathrm{g}^{-1}\end{array}$ & $\%$ & $\begin{array}{l}\mathrm{mg} \\
\mathrm{g}^{-1}\end{array}$ & & \\
\hline 14:0 & $1.7^{\mathrm{a}}$ & 8.5 & $3.4^{\mathrm{b}}$ & 21.3 & $3.6^{\mathrm{b}}$ & 23.7 & $3.8^{\mathrm{b}}$ & - & $3.3^{\mathrm{bc}}$ & 22.0 & $3.0^{\mathrm{b}}$ & 21.1 & 3.6 & 21.9 \\
\hline $15: 0$ & $1.0^{\mathrm{ab}}$ & 5.1 & $1.4^{\mathrm{c}}$ & 8.9 & $1.3^{b c}$ & 8.9 & $1.4^{\mathrm{c}}$ & - & $1.2^{\mathrm{bc}}$ & 8.1 & $0.8^{\mathrm{a}}$ & 6.1 & 0.9 & 5.5 \\
\hline $16: 0$ & $14.9^{\mathrm{a}}$ & 73.9 & $19.6^{\mathrm{bc}}$ & 120.6 & $19.7^{b c}$ & 131.4 & $20.9^{c}$ & - & $19.2^{\mathrm{bc}}$ & 130.4 & $17.9^{\mathrm{b}}$ & 125.6 & 19.8 & 121.5 \\
\hline $16: 1 n-7$ & $6.5^{\mathrm{a}}$ & 32.5 & $8.8^{\mathrm{ab}}$ & 54.8 & $9.9^{\mathrm{b}}$ & 65.9 & $10.1^{\mathrm{b}}$ & - & $11.2^{\mathrm{b}}$ & 74.2 & $8.6^{\mathrm{ab}}$ & 60.2 & 9.8 & 59.8 \\
\hline $17: 0$ & 1.3 & 6.7 & 1.8 & 11.0 & 1.5 & 10.2 & 1.6 & - & 1.3 & 9.6 & 1.2 & 8.4 & 1.2 & 7.5 \\
\hline $18: 0$ & $9.0^{\mathrm{b}}$ & 45.0 & $7.3^{\mathrm{a}}$ & 44.3 & $6.9^{\mathrm{a}}$ & 45.7 & $6.8^{\mathrm{a}}$ & - & $6.7^{\mathrm{a}}$ & 45.9 & $6.9^{\mathrm{a}}$ & 48.6 & 6.9 & 42.3 \\
\hline $18: 1 n-9$ & $11.2^{\mathrm{a}}$ & 55.6 & $11.2^{\mathrm{a}}$ & 68.1 & $11.5^{\mathrm{ab}}$ & 76.6 & $11.3^{\mathrm{a}}$ & - & $12.6^{\mathrm{b}}$ & 85.2 & $11.9^{\mathrm{ab}}$ & 83.1 & 11.3 & 69.6 \\
\hline $18: 1 n-7$ & $4.1^{\mathrm{a}}$ & 20.2 & $4.3^{\mathrm{ab}}$ & 26.8 & $4.4^{\mathrm{ab}}$ & 29.2 & $4.7^{b}$ & - & $4.1^{\mathrm{a}}$ & 28.3 & $4.4^{\mathrm{ab}}$ & 31.2 & 4.9 & 29.9 \\
\hline $20: 4 n-6$ & $8.7^{\mathrm{c}}$ & 43.1 & $4.7^{\mathrm{ab}}$ & 28.1 & $4.4^{\mathrm{a}}$ & 29.1 & $4.5^{\mathrm{a}}$ & - & $4.8^{\mathrm{ab}}$ & 33.1 & $5.3^{\mathrm{b}}$ & 37.6 & 4.3 & 26.1 \\
\hline $20: 5 n-3$ & $15.0^{\mathrm{b}}$ & 74.3 & $12.1^{\mathrm{ab}}$ & 72.2 & $8.0^{\mathrm{a}}$ & 76.3 & $10.8^{\mathrm{ab}}$ & - & $10.4^{\mathrm{ab}}$ & 72.4 & $14.1^{\mathrm{ab}}$ & 98.5 & 13.8 & 84.4 \\
\hline $22: 4 n-6$ & $1.0^{\mathrm{a}}$ & 4.8 & $0.9^{\mathrm{a}}$ & 5.6 & $1.2^{\mathrm{a}}$ & 7.8 & $1.0^{\mathrm{a}}$ & - & $1.7^{\mathrm{b}}$ & 11.6 & $0.8^{\mathrm{a}}$ & 6.0 & 0.9 & 5.2 \\
\hline $22: 5 n-6$ & $0.9^{b c}$ & 4.2 & $0.7^{\mathrm{a}}$ & 4.0 & $0.8^{\mathrm{ab}}$ & 5.1 & $0.6^{\mathrm{a}}$ & - & $1.1^{\mathrm{c}}$ & 7.0 & $0.7^{\mathrm{ab}}$ & 5.5 & 0.7 & 4.6 \\
\hline $22: 5 n-3$ & 2.1 & 10.2 & 1.8 & 10.9 & 2.1 & 13.8 & 1.9 & - & 2.4 & 16.1 & 1.9 & 13.1 & 1.8 & 11.1 \\
\hline $22: 6 n-3$ & 9.8 & 48.9 & 9.2 & 53.5 & 8.3 & 54.7 & 7.1 & - & 7.7 & 54.2 & 9.0 & 62.5 & 8.4 & 51.2 \\
\hline SFA & 29.7 & 148.0 & 33.9 & 207.8 & 36.9 & 222.6 & 34.8 & - & 33.1 & 226.0 & 30.5 & 213.7 & 33.9 & 207.5 \\
\hline MUFA & $23.6^{\mathrm{a}}$ & 117.9 & $26.9^{\mathrm{ab}}$ & 164.3 & $28.5^{\mathrm{bc}}$ & 190.1 & $28.8^{\mathrm{bc}}$ & - & $31.8^{\mathrm{c}}$ & 214.5 & $27.8^{\mathrm{b}}$ & 194.5 & 29.0 & 177.7 \\
\hline PUFA & $41.3^{b}$ & 204.8 & $33.7^{\mathrm{ab}}$ & 199.1 & $28.8^{\mathrm{a}}$ & 213.5 & $30.0^{\mathrm{a}}$ & - & $31.8^{\mathrm{ab}}$ & 220.1 & $36.2^{\mathrm{ab}}$ & 251.4 & 33.6 & 206.0 \\
\hline$n-3$ & 27.7 & 139.3 & 24.7 & 147.7 & 20.0 & 157.2 & 21.4 & - & 21.4 & 151.2 & 26.8 & 187.4 & 25.3 & 156.3 \\
\hline$n-6$ & $13.5^{\mathrm{c}}$ & 65.5 & $9.0^{\mathrm{a}}$ & 51.4 & $8.8^{\mathrm{a}}$ & 56.3 & $8.6^{\mathrm{a}}$ & - & $10.4^{\mathrm{b}}$ & 68.8 & $9.4^{\mathrm{ab}}$ & 63.9 & 8.3 & 49.7 \\
\hline $\begin{array}{l}n-3 \\
\text { HUFA }\end{array}$ & 27.6 & 135.5 & 23.9 & 138.7 & 19.1 & 147.6 & 20.4 & - & 21.2 & 145.6 & 25.9 & 177.3 & 25.0 & 150.4 \\
\hline $\begin{array}{l}n-3 / \\
n-6\end{array}$ & 2.06 & & 2.75 & & 2.23 & & 2.47 & & 2.07 & & 2.84 & & 3.05 & \\
\hline $\begin{array}{l}\text { DHA/ } \\
\text { EPA }\end{array}$ & 0.66 & & 0.75 & & 0.74 & & 0.66 & & 0.74 & & 0.64 & & 0.61 & \\
\hline
\end{tabular}

Data represent mean values. For clarity, a selection of FA $>1 \%$ is presented and standard deviations are omitted (variation was generally $<15 \%$ ). Sums include FA present in minor concentrations not listed in the table. Within a row, \% values with different superscript letters are significantly different $(P \leq 0.05)$.

Refer to the previous table for abbreviations. 
Table 6

Concentrations of vitamins $\mathrm{C}$ and $\mathrm{E}$ in ovaries of $L$. vannamei in different stages of sexual maturation, and in nauplii

\begin{tabular}{|c|c|c|c|c|c|c|c|}
\hline & \multicolumn{6}{|l|}{ Stage } & \multirow[t]{2}{*}{ Nauplii } \\
\hline & 0 & 1 & 2 & 3 & 4 & SF & \\
\hline \multicolumn{8}{|l|}{ Vitamin $C$} \\
\hline $\mathrm{AA}\left(\mathrm{mg} \mathrm{kg}^{-1}\right)$ & $397.7^{\mathrm{ab}}$ & $459.7^{\mathrm{b}}$ & $394.9^{\mathrm{ab}}$ & $351.6^{\mathrm{ab}}$ & $461.2^{\mathrm{b}}$ & $219.0^{\mathrm{a}}$ & 241.4 \\
\hline \multicolumn{8}{|l|}{ Vitamin E } \\
\hline $\begin{array}{l}\alpha \text {-Tocopherol } \\
\left(\mathrm{mg} \mathrm{kg}^{-1}\right)\end{array}$ & $67.6^{\mathrm{a}}$ & $325.8^{\mathrm{b}}$ & $370.5^{\mathrm{b}}$ & - & $374.4^{\mathrm{b}}$ & $124.4^{\mathrm{a}}$ & 392.9 \\
\hline $\begin{array}{l}\gamma \text {-Tocopherol } \\
\left(\mathrm{mg} \mathrm{kg}^{-1}\right)\end{array}$ & $0.00^{\mathrm{a}}$ & $1.63^{\mathrm{c}}$ & $2.50^{d}$ & - & $2.40^{\mathrm{d}}$ & $0.73^{b}$ & 4.17 \\
\hline
\end{tabular}

Data represent mean values. Within a row, values with different superscript letters are significantly different $(P \leq 0.05)$

Abbreviations: AA, ascorbic acid; SF, spent female.

remained stable. No major changes in the FA composition occurred after spawning. Naupliar FA composition reflected that of mature ovaries. None of the FA was incorporated preferentially into the nauplii, though the naupliar $n-3 / n-6$ ratio exceeded that for each of stage 0 and stage 4 ovaries. Additionally, the naupliar DHA/EPA ratio in the ovaries was lower than that of stage 1, 2 and 4 females.

\subsection{Vitamin content during ovarian maturation and after spawning}

Table 6 shows the AA concentrations in the ovaries for the different stages of sexual maturation and in nauplii. AA concentrations were not significantly different during ovarian maturation. After spawning, the AA content decreased significantly. The concentration of AA in nauplii was similar to that in ovaries of spent females. The concentration of AA in spent females was significantly lower than that in the ovaries of both stage 1 and stage 4 spawners.

Ovarian and naupliar vitamin E concentrations ( $\alpha$-tocopherol and $\gamma$-tocopherol) are presented in Table 6. $\alpha$-Tocopherol was present at much higher concentrations than $\gamma$-tocopherol. The levels of both forms increased significantly at the onset of ovarian maturation and $\gamma$-tocopherol continued to increase until stage 2 was reached. A significant decline at post-spawning was observed for both forms. High concentrations were recovered in the resulting nauplii. $\gamma$-Tocopherol content in nauplii was twofold higher than that in stage 4 ovaries.

\section{Discussion}

The increases in GSI and ovarian DM during sexual maturation indicate nutrient accumulation. In rapidly maturing L. vannamei, the accumulation may occur in as little 
as 3 days (Maturation Department, CENAIM-ESPOL Foundation, unpublished data), reflecting a daily increase of $0.5 \mathrm{~g}$ DW per ovary. Lipid accumulation contributes significantly to this increase in ovarian mass during the early stage of maturation (a 2.5-fold increase in ovarian TL from stage 0 to stage 1). An increase in ovarian TL was also observed in studies with other shrimp species; however, in contrast to our findings, the increase of TL in most of these species continued until the fully mature stage was reached, i.e. F. aztecus (Castille and Lawrence, 1989), F. duorarum (Gehring, 1974), Fen. indicus (Read and Caulton, 1980), M. japonicus (Teshima and Kanazawa, 1983; Teshima et al., 1989), Mel. kerathurus (Mourente and Rodriguez, 1991), L. setiferus (Castille and Lawrence, 1989), Ple. muelleri (Jeckel et al., 1989), P. monodon (Millamena and Pascual, 1990) and P. semisulcatus (Ravid et al., 1999). The observed decrease in TL of the midgut gland during the maturation process is similar to that found in the studies on Fen. indicus (Galois, 1984), M. japonicus (Teshima and Kanazawa, 1983; Teshima et al., 1989), Mel. kerathurus (Mourente and Rodriguez, 1991), L. setiferus (Castille and Lawrence, 1989) and P. monodon (Millamena and Pascual, 1990). However, in the present study, the decrease in midgut gland TL did not start at early maturation. Lipid accumulation in the ovaries occurred prior to the depletion of lipid in the midgut gland, thus contradicting the arguments of some of the previously cited authors who have concluded that the midgut gland is at the origin of the lipids accumulated in the ovaries. While the midgut gland is accurately identified as a major lipid storage and processing organ (Clarke, 1982; Teshima et al., 1989; Vogt et al., 1985), it seems that some of the ovarian lipids must be derived from either other body reserves, de novo synthesis or diet. Clarke (1982) and Castille and Lawrence (1989) similarly found that the amount of lipids accumulated in the ovaries of Chorismus antarcticus and $F$. aztecus, respectively, surpassed the amount stored in the midgut gland. Additionally, Clarke (1982) and Mourente (1996) demonstrated that the rate of de novo lipid synthesis in ovaries was too low to significantly contribute to the increase in ovarian TL. These findings lead to the conclusion that maturing shrimp are probably dependent on dietary lipids for normal lipid accumulation in the ovaries. Similar results were obtained with wild Mac. rosenbergii (Cavalli, 2000).

In the present study, a remarkable post-spawning reduction in the TL levels of the ovary was observed, indicating a major transfer of lipid to the offspring. Moreover, nauplii contained higher TL levels than fully developed ovaries.

The main LC of midgut gland lipids were FFA, TAG, PC and SE, with NL prevailing over POL. The broad features of these data are similar to those reported for $M$. japonicus (Teshima and Kanazawa, 1983). Our results suggest that the changes in midgut gland TL concentrations and POL/NL ratio are mainly due to a reduction in TAG, the principal lipids for energy storage. The concentration of $\mathrm{PC}$, the principal structural lipid did not vary appreciably. Other PL increased, probably as a result of the transformation of TAG to PL in the midgut gland followed by transport to the ovaries as water-soluble lipoproteins in the haemolymph (Chang and O'Connor, 1983; Teshima and Kanazawa, 1983; Ravid et al., 1999). FFA were present in the midgut gland at higher proportions than those in the ovaries, and increased moderately with sexual maturation. High proportions of FFA were also found in the midgut gland of $M$. japonicus (10.0-19.0\% in Teshima and Kanazawa, 1983) and in ovaries of Ple. 
muelleri (5.2-9.5\% in Jeckel et al., 1989), and therefore do not provide evidence of lipid deterioration.

PL and TAG were the most important lipids in the ovaries and have been reported to be the principle ovarian lipids in F. duorarum (Gehring, 1974), M. japonicus (Teshima and Kanazawa, 1983), Mel. kerathurus (Mourente and Rodriguez, 1991) and P. semisulcatus (Ravid et al., 1999). In Ple. muelleri (Jeckel et al., 1989), TAG, steryl sterols and FFA were dominant. PL are known to be important contributors to the structure of the ovarian membrane (Chang and O'Connor, 1983; Ravid et al., 1999). Changes in ovarian TL concentration as sexual maturation proceeds can be largely attributed to an increase in TAG. While egg glucose and TAG reserves are metabolized at a substantial rate to provide energy during egg development and hatching (Chang and O'Connor, 1983; Palacios et al., 1998, 1999), naupliar TAG percentages are still very high $(33.5 \%)$. This is an indicator of good quality, as nauplii with high energy stores have a greater chance of successful metamorphosis to the zoea stage (Lavens and Sorgeloos, 1991). With the exception of PC, phospholipid concentrations decrease slightly during maturation. These qualitative changes agree with the results reported by the aforementioned authors, with the exception of Teshima and Kanazawa (1983) who only found an increase in TAG.

As opposed to PA and PE, ovarian PC levels decreased significantly after the eggs were transferred out of the ovaries, which provides evidence of the importance of PC for embryological and larval development. The observed high naupliar PC levels as compared to other PL support the findings of D'Abramo et al. (1981) and Kanazawa (1993) who have stated that PC is the most active PL in marine fish and crustaceans. The positive effect of dietary PC on growth, survival and stress tolerance of shrimp larvae has been documented in several studies (for review see Coutteau et al., 1997). A close resemblance exists between the FA profile of $L$. vannamei tissues in the present study and those reported for other shrimp species. The same predominance of 16:00, 18:00, 16:1 $n-7,18: 1 n-9$, ARA, EPA and DHA was found in total lipids of ovaries and the midgut gland of reproductive L. setiferus (Middleditch et al., 1980), M. japonicus (Teshima and Kanazawa, 1983; Teshima et al., 1989), Mel. kerathurus (Mourente and Rodriguez, 1991) and Ple. muelleri (Jeckel et al., 1989). In contrast, $18: 1 n-7$ was not observed to be predominant in M. japonicus nor Ple. muelleri, while 20:1 $n-9$ was present in high proportions in the ovaries of M. japonicus, Mel. kerathurus and Ple. muelleri.

SFA and MUFA are abundant in detritus and in natural food organisms that are consumed by shrimp (Jeckel et al., 1989). In addition, these FA are synthesised by crustaceans during maturation (Clarke, 1982; Chang and O'Connor, 1983). This activity could explain the increase in 16:0, the principal end product of FA synthesis (Chang and O'Connor, 1983), and MUFA levels during early ovarian maturation in L. vannamei. The increase in 16:0 and MUFA could also reflect an increase in the proportion of NL in the ovarian lipids, as NL contain relatively more of these FA than POL.

Percentages of $n-3$ HUFA were comparatively high in all samples. HUFA either cannot be synthesised de novo (Kanazawa et al., 1977; Clarke, 1982; Chang and O'Connor, 1983) or may only be synthesised to a very limited extent through the conversion of 18:2n-6 and 18:3n-3 to $\mathrm{C}_{20}$ and $\mathrm{C}_{22}$ HUFA (Mourente, 1996). From 
the present study, it appears that diet is the origin of the HUFA in the midgut gland and ovaries of the spawners. Phytoplankton and zooplankton add substantial amounts of $n-3$ HUFA to the food web in marine ecosystems (Sargent et al., 1989; Napolitano, 1990). Successful maturation diets consisting of bivalves, squid and bloodworms are also rich sources of $n-3$ HUFA (Middleditch et al., 1980; Lytle et al., 1990). The importance of $n-3$ HUFA for crustacean maturation and reproduction has been recognized for many years (Harrison, 1990), and several studies with shrimp broodstock have noticed a good relationship with dietary $n-3$ HUFA content and better egg and larval quality (Xu et al., 1994; Cahu et al., 1995; Wouters et al., 1999).

As opposed to the findings of Mourente and Rodriguez (1991) with Mel. kerathurus, concentrations of $n-3$ HUFA in de midgut gland and ovaries did not increase during maturation in the present study, possibly indicating that they are not important for the maturation process itself. This finding is not supported by the results of Alava et al. (1993a) who demonstrated that ovarian maturation in M. japonicus was retarded when a HUFA-free diet was given, but agrees with the results of one of our previous studies (Wouters et al., 1999), in which the removal of the PUFA fraction in the enrichment product of the Artemia biomass supplement did not affect the maturation frequency in L. vannamei spawners. The importance of $n-3$ HUFA is probably more related to their role in larval development.

Noteworthy are the high levels of the ARA in both midgut gland and ovaries. ARA is thought to be a precursor in the synthesis of prostaglandins, which plays a role in the reproduction of mammals and certain fish and insect species (Sargent et al., 1989). Nevertheless, the decrease of ARA in the ovaries during early maturation demonstrates that this FA is not deposited preferentially into the ovaries, contradicting the theory that it is necessary for shrimp maturation (Middleditch et al., 1980). Maybe ARA is only required at moderate dietary levels, as suggested by Lytle et al. (1990). These authors further contend that a delicate balance exists between $n-3$ and $n-6$ fatty acids. In the present study, $n-3 / n-6$ ratios are high in both midgut gland and ovaries. As nauplii displayed a higher $n-3 / n-6$ ratio as ovaries, i.e. $3: 1, n-3$ FA were preferentially transferred to the offspring. This suggests that $n-3$ FA have a higher value than $n-6$ FA for L. vannamei broodstock animals and larvae, in agreement with the observations made on the FA requirements of Fen. chinensis juveniles (Xu et al., 1993, 1994).

The observed concentrations of vitamin $\mathrm{C}$, a water-soluble component, in the ovaries of immature, maturing and mature ovaries were high (ranging from 351.6 to $461.2 \mathrm{mg}$ $\mathrm{kg}^{-1}$ ). In this respect, our results contrast with the ovarian AA concentrations of 6-18 $\mathrm{mg} \mathrm{kg}^{-1}$ reported by Sangha et al. (1998a,b) for captive L. vannamei spawners. As this vitamin cannot be synthesised by shrimp, differences should be due to differences in dietary levels of vitamin C. However, even after adding $3000 \mathrm{mg} \mathrm{kg}^{-1}$ L-ascorbyl-2polyphosphate to the diet, Sangha et al. could not detect ovarian AA levels above $93 \mathrm{mg}$ $\mathrm{kg}^{-1}$. In the present study, AA concentrations in the ovaries of spent females and nauplii were about twofold lower than that in mature ovaries, suggesting that AA was used during egg development and hatching. Cavalli (2000) determined ovarian AA levels of 210 to $450 \mathrm{mg} \mathrm{kg}^{-1}$ in wild Mac. rosenbergii and provide evidence of AA consumption by developing embryos. Cahu et al. (1995) also determined AA concentrations of $197-523 \mathrm{mg} \mathrm{kg}^{-1}$ in the eggs of Fen. indicus and found that high dietary AA 
induced better hatching. They suggested that the beneficial effect of AA might be associated with its action in the hydroxylation of protein-bound proline and lysine, which provide stable triple helicoidal collagen through the embryonic stages.

The increase in concentrations of vitamin $\mathrm{E}$ in the ovary with proceeding sexual maturation of $L$. vannamei suggests that this vitamin plays a critical role in the shrimp maturation process. This observation is supported by findings of Alava et al. (1993b) who delayed ovarian maturation in $M$. japonicus by feeding them a diet without supplementary vitamin $\mathrm{E}$. The levels of $\alpha$-tocopherol in mature ovaries $\left(374.4 \mathrm{mg} \mathrm{kg}^{-1}\right)$ and nauplii (392.9 $\mathrm{mg} \mathrm{kg}^{-1}$ ) are within the range of those reported by Cahu et al. (1995) for Fen. indicus eggs obtained under captive conditions with domesticated animals (177-587 $\mathrm{mg} \mathrm{kg}^{-1}$ ), and those reported by Cavalli (2000) in the ovaries of wild Mac. rosenbergii (143-425 $\left.\mathrm{mg} \mathrm{kg}^{-1}\right)$. The post-spawning reduction in the ovaries and subsequent recovery of high levels in the nauplii provide further evidence of the importance of this lipid soluble vitamin for larval development. Egg hatching rate and egg $\alpha$-tocopherol concentrations of Fen. indicus were also positively correlated in the studies conducted by Cahu et al. (1991, 1995). In the present study, the $\alpha$-tocopherol and $\gamma$-tocopherol reserves were not depleted during embryonic development and/or hatching, probably because they are constituents of the cell membrane. Another function of AA and $\alpha$-tocopherol in solution, membranes and lipoproteins is mentioned by Niki et al. (1995). AA and $\alpha$-tocopherol act as potent hydrophilic and lipophilic antioxidants, respectively, protecting the cell constituents by scavenging radicals. Furthermore, AA reduces the resulting $\alpha$-tocopherol radical efficiently to regenerate $\alpha$-tocopherol. This relationship may be an additional reason why AA levels are low and $\alpha$-tocopherol levels are high in nauplii.

\section{Acknowledgements}

The authors wish to thank Yela Paredes and Luis Gómez for their assistance with the sampling of the animals. The technical support of Karla Van Ryckeghem, Geert Vande Wiele, Marie Claire Dereu, Anita Dehaese, Christ Mathieu, Huo Jizheng and Pascale Hoste is also highly appreciated. This work was supported by the VLIR-Own Initiative Programme of the Flemish Inter-University Council (V.L.I.R.).

\section{References}

Alava, V.R., Kanazawa, A., Teshima, S.I., Koshio, S., 1993a. Effect of dietary vitamins A, E and C on the ovarian development of Penaeus japonicus. Nippon Suisan Gakkaishi 59 (7), 1235-1241.

Alava, V.R., Kanazawa, A., Teshima, S.I., Koshio, S., 1993b. Effect of dietary phospholipids and $n-3$ highly unsaturated fatty acids on ovarian development of Kuruma prawn. Nippon Suisan Gakkaishi 59 (7), $345-351$.

Bray, W.A., Lawrence, A.L., Lester, L.J., 1990. Reproduction of eyestalk-ablated Penaeus stylirostris fed various levels of total dietary lipid. J. World Aquacult. Soc. 21, 41-52. 
Cahu, C., Fakhfakh, M., Quazuguel, P., 1991. Effect of dietary $\alpha$-tocopherol level on reproduction of Penaeus indicus. In: Lavens, P., Sorgeloos, P., Jaspers, E., Ollevier, F. (Eds.), LARVI '91-Fish and Crustacean Larviculture Symposium, 1991, Gent, Belgium. European Aquaculture Society Special Publication No. 15, pp. 242-244.

Cahu, C.L., Cuzon, G., Quazuguel, P., 1995. Effect of highly unsaturated fatty acids, alpha-tocopherol and ascorbic acid in broodstock diet on egg composition and development of Penaeus indicus. Comp. Biochem. Physiol. 112, 417-424.

Castille, F.L., Lawrence, A.L., 1989. Relationship between maturation and biochemical composition of the gonads and digestive glands of the shrimps Penaeus aztecus Ives and Penaeus setiferus (L.). J. Crustacean Biol. 9, 202-211.

Castell, D.J., Boston, L.D., Miller, R.J., Kenchington, T., 1995. The potential identification of the geographic origin of lobster eggs from various wild stocks based on fatty acid composition. Can. J. Fish. Aquat. Sci. $52,1135-1140$.

Cavalli, R.O., 2000. Broodstock nutrition and offspring quality of the freshwater prawn Macrobrachium rosenbergii (de Man). PhD dissertation. Faculty of agricultural and applied biological sciences, Ghent University, Gent, Belgium.

Chamberlain, G.W., 1988. Stepwise investigation of environmental and nutritional requirements for reproduction of penaeid shrimp. PhD dissertation, Department Wildlife and Fisheries Sciences, Texas A\&M University, TX, USA

Chang, E., O’Connor, J., 1983. Metabolism and transport of carbohydrates and lipids. In: Mantel, H. (Ed.), The Biology of Crustacea, vol. 5, Academic Press, New York, pp. 263-287.

Clarke, A., 1982. Lipid synthesis and reproduction in the polar shrimp Chorismus antarticus. Mar. Ecol. Prog. Ser. 9, 81-90

Coutteau, P., Sorgeloos, P., 1995. Intercalibration exercise on the qualitative and quantitative analysis of fatty acids in Artemia and marine samples. ICES 211, ICES Coop. Res. Rep., 30 pp.

Coutteau, P., Geurden, I., Camara, M.R., Bergot, P., Sorgeloos, P., 1997. Review on the dietary effects of phospholipids in fish and crustacean larviculture. Aquaculture 155, 149-164.

D’Abramo, L.R., 1997. Triacylglycerol and fatty acids. In: D’Abramo, L.R., Conklin, D.E., Akiyama, D.M. (Eds.), Crustacean Nutrition, vol. 6, World Aquaculture Society, Baton Rouge, LA, pp. 71-84.

D’Abramo, L.R., Bordner, C.E., Conklin, D.E., Baum, N.A., 1981. Essentiality of dietary phophatidylcholine for the survival of juvenile lobsters. J. Nutr. 111, 425-431.

Folch, J., Lees, M., Sloane-Stanley, G.H., 1957. A simple method for the isolation and purification of total lipides from animal tissues. J. Biol. Chem. 266, 497-509.

Galois, R.G., 1984. Variations de la composition lipidique tissulaire au cours de la vitellogenese chez la crevette Penaeus indicus Milne Edwards. J. Exp. Mar. Biol. Ecol. 84, 155-166.

Gehring, W.R., 1974. Maturational changes in the ovarian lipid spectrum of the pink shrimp Penaeus duorarum Burkenroad. Comp. Biochem. Physiol. 49A, 511-524.

Harrison, K.E., 1990. The role of nutrition in maturation, reproduction and embryonic development of decapod crustaceans: a review. J. Shellfish Res. 9, 1-28.

Harrison, K.E., 1997. Broodstock nutrition and maturation diets. In: D’Abramo, L.R., Conklin, D.E., Akiyama, D.M. (Eds.), Crustacean Nutrition, vol. 6, The World Aquaculture Society, Baton Rouge, LA, pp. 390-408.

Huo, J.Z., Nelis, H.J., Lavens, P., Sorgeloos, P., De Leenheer, A.P., 1999. Simultaneous determination of $\alpha$-tocopherol acetate and tocopherols in aquatic organisms and fish feed. J. Chromatogr. B 724, 249-255.

Jeckel, W.H., Aizpun de Moreno, J.E., Moreno, V.J., 1989. Biochemical composition, lipid classes and fatty acids in the ovary of the shrimp Pleoticus muelleri Bate. Comp. Biochem. Physiol. 92B, 271-276.

Kanazawa, A., 1993. Essential phospholipids of fish and crustaceans. In: Kaushik, S.F., Luquet, P. (Eds.), Fish Nutrition in Practice, 1993. Ed. INRA, Paris Les Colloques nr. 61, Biarritz (France), pp. 519-530.

Kanazawa, A., Teshima, S.I., Tokiwa, S., 1977. Nutritional requirements of prawn: VII. Effect of dietary lipids on growth. Bull. Jpn. Soc. Sci. Fish. 43, 849-856.

Lavens, P., Sorgeloos, P., 1991. Variation in egg and larval quality in various fish and crustacean species. In: Lavens, P., Sorgeloos, P., Jaspers, E., Ollevier, F. (Eds.), LARVI '91-Fish and Crustacean Larviculture Symposium, 1991, Gent, Belgium. European Aquaculture Society Special Publication No. 15, pp. $221-222$. 
Lytle, J.S., Lytle, T.F., Ogle, J.T., 1990. Polyunsaturated fatty acid profiles as a comparative tool in assessing maturation diets of Penaeus vannamei. Aquaculture 89, 287-299.

Mead, R., Curnow, R.N., Hasted, A.M., 1993. Statistical Methods in Agriculture and Experimental Biology. Chapman \& Hall, London, UK, 415 pp.

Middleditch, B.S., Missler, S.R., Hines, H.B., McVey, J.B., Brown, A., Ward, D.G., Lawrence, A.L., 1980. Metabolic profiles of penaeid shrimp: dietary lipids and ovarian maturation. J. Chromatogr. 195, 359-368.

Millamena, O.M., Pascual, F.P., 1990. Tissue lipid content and fatty acid composition of Penaeus monodon Fabricius broodstock from the wild. J. World Aquacult. Soc. 21, 116-121.

Montaño, M., Navarro, J.C., 1996. Fatty acids of wild and cultured Penaeus vannamei larvae from Ecuador. Aquaculture 142, 259-268.

Mosquera, G., 1999. La pesquería artesanal de reproductores de camarón: Estado actual y sus perspectivas. In: Rosero, J.Z., Burgos, M. (Eds.). Principios y criterios técnicos para el desarrollo sustentable de la pesquería de reproductores de camarón. Informe técnico del Instituto Nacional de Pesca y el Programa de Manejo de Recursos Costeros, Guayaquil, Ecuador, pp. 1-31.

Mourente, G., 1996. In vitro metabolism of C-14-polyunsaturated fatty acids in midgut gland and ovary cells from Penaeus kerathurus Forskal at the beginning of sexual maturation. Comp. Biochem. Physiol. 115B, 255-266.

Mourente, G., Rodriguez, A., 1991. Variation in the lipid content of wild-caught females of the marine shrimp Penaeus kerathurus during sexual maturation. Mar. Biol. 110, 21-28.

Napolitano, G.E., 1990. Fatty acid composition of three cultured algal species (Isochrisis galbana, Chaetoceros gracilis and Chaetoceros calcitrans) used as food for bivalve larvae. J. World Aquacult. Soc. 21, $122-130$.

Nelis, H.J., De Leenheer, A.P., Merchie, G., Lavens, P., Sorgeloos, P., 1997. Liquid chromatographic determination of vitamin $\mathrm{C}$ in aquatic organisms. J. Chromatogr. Sci. 35, 337-341.

Niki, E., Noguchi, N., Tsuchihashi, H., Gotoh, N., 1995. Interaction among vitamin C, vitamin E, and $\beta$-carotene. Am. J. Clin. Nutr. 62, 1322S-1326S (Suppl.).

Olsen, R.E., Henderson, R.J., 1989. The rapid analysis of neutral and polar marine lipids using double-development HPTLC and scanning densitometry. J. Exp. Mar. Biol. Ecol. 129, 197-198.

Palacios, E., Ibarra, A.M., Ramirez, J.L., Portillo, G., Racotta, I.S., 1998. Biochemical composition of eggs and nauplii in white Pacific shrimp, Penaeus vannamei (Boone), in relation to the physiological condition of spawners in a commercial hatchery. Aquacult. Res. 29, 183-189.

Palacios, E., Perez-Rostro, C.I., Ramirez, J.L., Ibarra, A.M., Racotta, I.S., 1999. Reproductive exhaustion in shrimp (Penaeus vannamei) reflected in larval biochemical composition, survival and growth. Aquaculture 171, 309-321.

Ravid, T., Tietz, A., Khayat, M., Boehm, E., Michelis, R., Lubzens, E., 1999. Lipid accumulation in the ovaries of a marine shrimp Penaeus semisulcatus (De Haan). J. Exp. Biol. 202, 1819-1829.

Read, G.H.L., Caulton, M.S., 1980. Changes in mass and chemical composition during the molt cycle and ovarian development in inmature and mature Penaeus indicus Milne Edwards. Comp. Biochem. Physiol. 66A, 431-437.

Sangha, R.S., Chavez-Sanchez, M.C., Martinez-Palacios, C.A., Martinez-Rodriguez, I.E., 1998a. Supplementing L-ascorbic-2-polyphosphate in broodstock diet of Penaeus (Litopenaeus) vannamei at a hatchery. Book of Abstracts, Aquaculture '98 Confrence of the World Aquaculture Society, 1998. Bally's, Las Vegas, NV, USA, p. 672.

Sangha, R.S., Chavez-Sanchez, M.C., Martinez-Rodriguez, I.E., Martinez-Palacios, C.A., 1998b. Changes in total ascorbic acid levels in the ovaries and midgut gland in broodstock Penaeus (Litopenaeus) vannamei: before and after spawns. Book of Abstracts, Aquaculture '98 Confrence of the World Aquaculture Society, 1998. Bally's, Las Vegas, NV, USA, p. 673.

Sargent, J., Henderson, R.J., Tocher, D.R., 1989. The lipids. In: Halver, J.E. (Ed.), Fish Nutrition. Academic Press, London, UK, pp. 153-219.

Teshima, S.I., Kanazawa, A., 1983. Variation in lipid composition during the ovarian maturation of the prawn. Bull. Jpn. Soc. Sci. Fish. 49, 957-962.

Teshima, S.I., Kanazawa, A., Koshio, S., Horinouchi, K., 1989. Lipid metabolism of the prawn Penaeus japonicus during maturation: variation in lipid profiles of the ovary and hepatopancreas. Comp. Biochem. Physiol. 92B, 45-49. 
Vogt, G., Storch, V., Quinitio, E.T., Pascual, F.P., 1985. Midgut gland as monitor organ for the nutritional value of diets in Penaeus monodon (Decapoda). Aquaculture 48, 1-12.

Ways, P., Hanahan, D.J., 1964. Characterization and quantification of red cell lipids in normal man. J. Lipid Res. 5, 318-328.

Wouters, R., Gómez, L., Lavens, P., Calderón, J., 1999. Feeding enriched Artemia biomassa to Penaeus vannamei broodstock: its effect on reproductive performance and larval quality. J. Shellfish Res. 18, $651-656$.

Xu, X.L., Ji, W., Castell, J.D., O’Dor, R.K., 1993. The nutritional value of dietary $n-3$ and $n-6$ fatty acids for the Chinese prawn (Penaeus chinensis). Aquaculture 118, 277-285.

Xu, X.L., Ji, W., Castell, J.D., O’Dor, R.K., 1994. Essential fatty acid requirements of the Chinese prawn, Penaeus chinensis. Aquaculture 127, 29-40. 